\title{
SHARP SLOPE BOUNDS FOR SWEEPING FAMILIES OF TRIGONAL CURVES
}

\author{
Anand Deopurkar And Anand Patel
}

\begin{abstract}
We establish sharp bounds for the slopes of curves in $\bar{M}_{g}$ that sweep out the locus of trigonal curves, reproving Stankova-Frenkel's bound of $7+6 / g$ for even $g$ and obtaining the bound $7+20 /(3 g+1)$ for odd $g$. For even $g$, we find an explicit expression of the so-called Maroni divisor in the Picard group of the space of admissible triple covers. For odd $g$, we describe the analogous extremal effective divisor and give a similar explicit expression.
\end{abstract}

\section{Introduction}

The seminal papers of Harris and Mumford [12] and Eisenbud and Harris [9] prove that the moduli space $\bar{M}_{g}$ of Deligne-Mumford stable curves of genus $g$ is of general type for $g \geq 23$. This result marks the beginning of the quest for describing the log-canonical models of $\bar{M}_{g}$, namely the spaces

$$
\bar{M}_{g}(\alpha)=\operatorname{Proj} \bigoplus_{n \geq 0} H^{0}\left(\overline{\mathcal{M}}_{g}, n\left(K_{\overline{\mathcal{M}}_{g}}+\alpha \delta\right)\right) .
$$

Understanding these spaces and the maps between them is an active area of research with important contributions by Hassett, Hyeon, Keel, Morrison and several others [11].

As a step towards this goal, it is important to understand the stable base loci of the linear systems $\left|K_{\overline{\mathcal{M}}_{g}}+\alpha \delta\right|$, or equivalently the linear systems $|s \lambda-\delta|$, where $\lambda$ is the Hodge class, $\delta$ the total boundary class and $s=13 /(2-\alpha)$. To test whether a given $X \subset \bar{M}_{g}$ lies in such a base locus, we may cover it by curves of high slope $(\delta / \lambda)$; if $X$ can be covered by curves of slope $s_{0}$ then it must be in the base locus of $|s \lambda-\delta|$ for $s<s_{0}$. We are thus led to the following question.

Question 1.1. For a subvariety $X \subset \bar{M}_{g}$, what is the highest s such that $X$ is covered by curves of slope $s$ ?

The first result in this direction came from the work of Cornalba and Harris [6, Section $4 \mathrm{~b}]$. They answered it for the locus of hyperelliptic curves, getting the slope bound $8+4 / g$. Next, Stankova-Frenkel [15, Theorems III and IV] found that the answer for the locus of trigonal curves is $7+6 / g$ if $g$ is even. More recently, Barja and Stoppino [2, Theorem 1.6] and Fedorchuk and Jensen [10, Theorem 1.2, Corollary 3.2] extended the bound to cover more general trigonal fibrations of even genus. Question 1.1 is particularly interesting for the loci of Brill-Noether special curves in

Received by the editors November 19, 2012.

1991 Mathematics Subject Classification. xxx. 
$\bar{M}_{g}$ because these loci are expected to appear in the base loci of the log-canonical linear systems.

In this paper, we settle the question for the locus of trigonal curves of any genus. We reprove the $7+6 / g$ bound for even $g$ and obtain the bound $7+20 /(3 g+1)$ for odd $g$.

Theorem 1.2 (Corollaries 5.4 and 5.8 in the main text). Let $g \geq 4$. Denote by $\bar{T}_{g}$ the closure in $\bar{M}_{g}$ of the locus of smooth trigonal curves. Set

$$
s_{g}= \begin{cases}7+6 / g & \text { if } g \text { is even, } \\ 7+20 /(3 g+1) & \text { if } g \text { is odd }\end{cases}
$$

Then,

(1) $\bar{T}_{g}$ is covered by curves of slope $s_{g}$.

(2) If $B \subset \bar{T}_{g}$ is a curve passing through a general point of $\bar{T}_{g}$, then its slope is at most $s_{g}$. In particular, $\bar{T}_{g}$ cannot be covered by curves of higher slope.

The word "general" in the second statement means away from some effective divisors, discussed below, whose classes are given explicitly in Propositions 5.3 and 5.7.

Our approach is classical, but we use a modern ingredient to make it feasible. For (1), we explicitly construct curves with slope $s_{g}$ that sweep out $\bar{T}_{g}$. For $(2)$, we explicitly construct an effective divisor $D \subset \bar{T}_{g}$ such that

$$
a[D]=s_{g} \lambda-\delta-\sum_{i} a_{i} \delta_{i},
$$

with $a>0$ and $a_{i} \geq 0$. It follows that any curve $B \subset \bar{T}_{g}$ whose generic point avoids $D$ and the boundary divisors $\delta_{i}$ must have $\left(s_{g} \lambda-\delta\right) \cdot B \geq 0$, yielding the bound in (1.2). For even $g$, the divisor $D$ is the closure of the locus of smooth trigonal curves that embed in an unbalanced scroll. For odd $g$, it is the closure of the locus of smooth trigonal curves that embed in $\mathbf{F}_{1}$ and are tangent to the directrix. Thus, an equivalent formulation of our result is that $\left(s_{g} \lambda-\delta\right)$ spans an edge of the effective cone in the $\langle\lambda, \delta\rangle$ plane in the Picard group of $\bar{T}_{g}$.

Here is the road-map for the proof. In Section 2, we describe a compactification of $T_{g}$ using admissible covers which is more convenient than $\bar{T}_{g}$. In Section 3 , we express the divisor classes $\lambda, \kappa$, and $\delta$ for a family of triple covers in terms of the linear algebraic data that defines the triple cover. In Section 4, we finish off Theorem 1.2 (1) using the linear algebraic description. This section does not require the more delicate orbifold considerations in the previous sections. In Section 5, we prove Theorem 1.2 (2) by computing the divisor class of $D$ in the Picard group of the admissible cover compactification.

We would like to highlight the modern ingredient that makes our approach successful. To compute the divisor class of $D$, we use test-curves that intersect the boundary divisors at generic points. This lets us avoid having to identify the points of the boundary that lie in $D$. Constructing enough such test-curves, however, is challenging. The basis of our trigonal constructions - the linear algebraic description — fails for admissible covers, which are not necessarily flat. The idea that rescues us is the formulation of admissible covers as flat covers of orbi-nodal curves due to Abramovich et 
al. [1]. Another piece of modern technology, namely the Grothendieck-Riemann-Roch theorem for Deligne-Mumford stacks [8] makes this computationally feasible.

We work over an algebraically closed field $k$ of characteristic zero. Genus always means arithmetic genus. By the genus of an orbi-curve, we mean the genus of its coarse space. A cover is a representable, finite, flat morphism. A triple cover $\phi: C \rightarrow \mathbf{P}^{1}$ is balanced (resp. unbalanced) if the degrees of the two summands of the vector bundle $\phi_{*} O_{C} / O_{\mathbf{P}^{1}}$ differ by at most 1 (resp. at least 2). Projectivizations are spaces of onedimensional quotients, following Grothendieck's convention. Throughout, $\zeta_{n}$ denotes a primitive $n$th root of unity.

\section{A compactification of $T_{g}$ and its boundary}

Let $\overline{\mathcal{M}}_{g}$ be the moduli stack of Deligne-Mumford stable curves of genus $g$. Let $\overline{\mathcal{H}}_{g}^{3}$ be the moduli stack of twisted admissible covers of genus $g$ and degree three with unordered branch points where at most two branch points are allowed to coincide. In symbols

$$
\overline{\mathcal{H}}_{g}^{3}=\{(\phi: \mathcal{C} \rightarrow \mathcal{P}, \mathcal{P} \rightarrow P)\}
$$

where

$P$ is a connected nodal curve of genus zero,

$\mathcal{P}$ is a balanced orbi-nodal curve in the sense of [1] with coarse space $\mathcal{P} \rightarrow P$,

$\mathcal{C}$ is a connected orbi-nodal curve of genus $g$,

$\phi: \mathcal{C} \rightarrow \mathcal{P}$ is a twisted admissible cover of degree 3 in the sense of [1],

$(P, \operatorname{br} \phi)$ is a $w$-stable pointed curve in the sense of $[13]$ with $w=\left(\frac{1}{2}, \ldots, \frac{1}{2}\right)$.

For the convenience of the reader, we briefly recall the meanings of some of the terms. A balanced orbi-nodal curve $\mathcal{P} \rightarrow P$ is a modification of $P$ at the nodes; étale locally around the nodes, $\mathcal{P} \rightarrow P$ has the form

$$
\left[(\operatorname{Spec} k[u, v] / u v) / \mu_{n}\right] \rightarrow \operatorname{Spec} k[x, y] / x y,
$$

where a generator $\zeta_{n}$ of $\mu_{n}$ acts by $\zeta_{n}: u \mapsto \zeta_{n} u$ and $\zeta_{n}: v \mapsto \zeta_{n}^{-1} v$. A twisted admissible cover $\phi: \mathcal{C} \rightarrow \mathcal{P}$ is a representable, flat, finite morphism of degree $d$, étale over the nodes and the generic points of the components of $\mathcal{P}$ such that the associated map $\mathcal{P} \backslash$ br $\phi \rightarrow B \mathbf{S}_{d}$ is representable. The last condition pins down the groups $\mu_{n}$ that define the stack structure on $\mathcal{P}$. Note that the induced map on the coarse spaces $\phi: C \rightarrow P$ is an admissible cover in the sense of Harris and Mumford $\left[12\right.$, Section 4]. Finally, the $w$-stability condition means that mult ${ }_{p}$ br $\phi \leq 2$ for all $p \in P$, and $\omega_{P}\left(\frac{\mathrm{br} \phi}{2}\right)$ is ample.

By standard methods, one can prove that $\overline{\mathcal{H}}_{g}^{3}$ is a connected, smooth, proper, Deligne-Mumford stack with a projective coarse space. We refer the reader to [7] for the technical details. The compactification $\overline{\mathcal{H}}_{g}^{3}$ and the more customary compactification by twisted admissible covers (where the branch points are distinct) are isomorphic on the level of coarse spaces. The functorial description that allows two branch points to collide, however, is quite convenient.

The open locus $\mathcal{H}_{g}^{3}$ where $P$ is smooth and br $\phi$ is reduced is the locus of simply branched trigonal curves. When two branch points coincide, $\mathcal{C}$ may develop either a node or a triple ramification point. In any case, the coarse space of $\mathcal{C}$ is at worst 
(a)

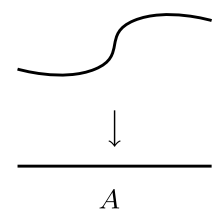

(d)

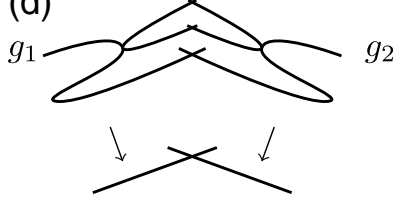

$\Delta_{1}\left(g_{1}, g_{2}\right)$

with $g_{1}+g_{2}=g-2$ and $g_{i} \geq 0$.

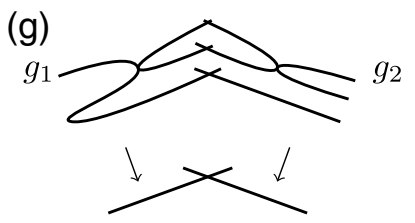

$\Delta_{4}\left(g_{1}, g_{2}\right)$

with $g_{1}+g_{2}=g-1$ and $g_{2} \geq 1$ (b)

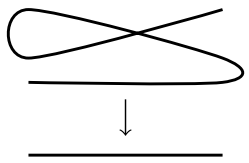

$\Delta$

(e)

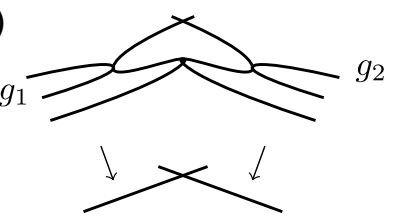

$\Delta_{2}\left(g_{1}, g_{2}\right)$

with $g_{1}+g_{2}=g-1$ and $g_{i} \geq 0$

(h)

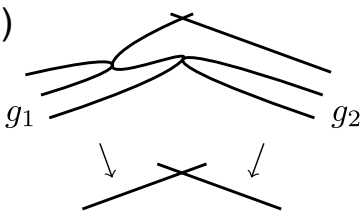

$\Delta_{5}\left(g_{1}, g_{2}\right)$

with $g_{1}+g_{2}=g$ and $g_{2} \geq 1$ (c)

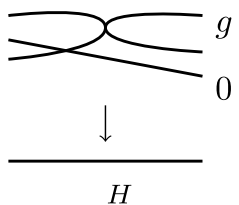

(f)

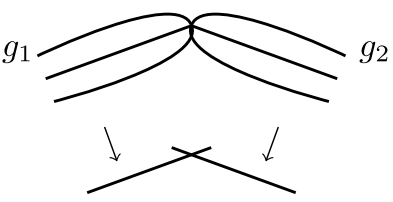

$\Delta_{3}\left(g_{1}, g_{2}\right)$

with $g_{1}+g_{2}=g$ and $g_{i} \geq 1$

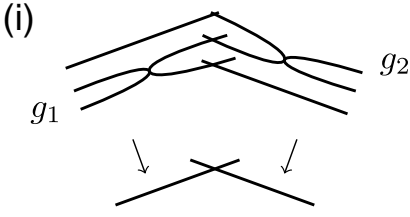

$\Delta_{6}\left(g_{1}, g_{2}\right)$

with $g_{1}+g_{2}=g$ and $g_{i} \geq 1$

Figure 1. Boundary divisors of $\overline{\mathcal{H}}_{g}^{3}$. (a) $A$, (b) $\Delta$, (c) $H$, (d) $\Delta_{1}\left(g_{1}, g_{2}\right)$ with $g_{1}+g_{2}=g-2$ and $g_{i} \geq 0$., (e) $\Delta_{2}\left(g_{1}, g_{2}\right)$ with $g_{1}+g_{2}=g-1$ and $g_{i} \geq 0$, (f) $\Delta_{3}\left(g_{1}, g_{2}\right)$ with $g_{1}+g_{2}=g$ and $g_{i} \geq 1$, (g) $\Delta_{4}\left(g_{1}, g_{2}\right)$ with $g_{1}+g_{2}=g-1$ and $g_{2} \geq 1$, (h) $\Delta_{5}\left(g_{1}, g_{2}\right)$ with $g_{1}+g_{2}=g$ and $g_{2} \geq 1$, (i) $\Delta_{6}\left(g_{1}, g_{2}\right)$ with $g_{1}+g_{2}=g$ and $g_{i} \geq 1$

a nodal curve of genus $g$. By contracting unstable rational tails, we get a morphism $\overline{\mathcal{H}}_{g}^{3} \rightarrow \overline{\mathcal{M}}_{g}$. We denote by $\lambda$ the Hodge line bundle and by $\delta$ the total boundary divisor of $\overline{\mathcal{M}}_{g}$. Abusing notation, we denote their pullbacks to $\overline{\mathcal{H}}_{g}^{3}$ by the same letters.

The boundary $\overline{\mathcal{H}}_{g}^{3} \backslash \mathcal{H}_{g}^{3}$ is a union of several divisors which we pictorially list in Figure 1. For clarity, we draw the admissible cover $C \rightarrow P$ instead of the twisted admissible cover $\mathcal{C} \rightarrow \mathcal{P}$.

The pictures are, of course, symbolic and only show the generic points. They are to be read as follows. A generic point of $A$ represents a trigonal curve with a triple ramification point. A generic point of $\Delta$ represents an irreducible trigonal curve with a node. A generic point of $H$ represents a hyperelliptic curve of genus $g$ with a rational tail attached at a non-ramification point. In the rest of the boundary divisors, the base is reducible. A generic point of $\Delta_{3}\left(g_{1}, g_{2}\right)$ represents two irreducible trigonal curves of genus $g_{1}$ and $g_{2}$ glued at a triple ramification point; this union maps to a union of two $\mathbf{P}^{1}$ s glued at a point. Likewise, a generic point of $\Delta_{5}\left(g_{1}, g_{2}\right)$ represents an irreducible trigonal curve of genus $g_{1}$ attached to a $\mathbf{P}^{1}$ at a non-ramification point and to a hyperelliptic curve of genus $g_{2}$ at a simple ramification point; this maps to a union of two $\mathbf{P}^{1} \mathrm{~s}$ glued at a point as shown in the diagram. We leave the interpretations of the 
other pictures to the reader. The order of $g_{1}$ and $g_{2}$ is important only in $\Delta_{4}\left(g_{1}, g_{2}\right)$ and $\Delta_{5}\left(g_{1}, g_{2}\right)$.

We often call the divisors $\Delta_{i}\left(g_{1}, g_{2}\right)$ (for $\left.1 \leq i \leq 6\right)$ and $H$ higher boundary divisors.

Proposition 2.1. The classes $\lambda, \delta$, and the higher boundary divisors $H, \Delta_{i}\left(g_{1}, g_{2}\right)$ for $1 \leq i \leq 6$ as shown in Figure 1 form a basis of $\operatorname{Pic}_{\mathbf{Q}}\left(\overline{\mathcal{H}}_{g}^{3}\right)$.

Proof. We know that $\operatorname{Pic}_{\mathbf{Q}}\left(\mathcal{H}_{g}^{3}\right)$ is trivial, for example from [15, Proposition 12.1] or [4, Theorem 1.1]. Hence, $\operatorname{Pic}_{\mathbf{Q}}\left(\overline{\mathcal{H}}_{g}^{3}\right)$ is generated by $A, \delta$ and the higher boundary divisors. By test-curve calculations (for example, those in Section 5), one can verify that $A, \delta$, and the higher boundary divisors are linearly independent and that one can use $\lambda$ instead of $A$.

\section{Evaluating the divisor classes on a family of triple covers}

The goal of this section is to compute the divisor classes $\lambda$ and $\delta$ associated with a family of triple covers in terms of the linear algebraic data that defines the triple cover (Proposition 3.1). We begin by recalling the definitions of $\lambda$ and $\delta$ for orbi-nodal curves and the linear algebraic description of triple covers.

Let $B$ be a smooth projective curve and $\pi: \mathcal{S} \rightarrow B$ a generically smooth, proper family of orbi-nodal curves. Denote by $\omega_{\mathcal{S} / B}$ the relative canonical bundle. Set

$$
\kappa_{\mathcal{S} / B}=\pi_{*}\left(c_{1}\left(\omega_{\mathcal{S} / B}\right)^{2}\right) \text {, and } \lambda_{\mathcal{S} / B}=c_{1}\left(R \pi_{*} O_{\mathcal{S}}\right) .
$$

We are interested in these classes only up to numerical equivalence. Denote by $\delta_{\mathcal{S} / B}$ the degree of the singular locus of $\mathcal{S} \rightarrow B$ interpreted in the orbifold sense, that is, so that the orbi-node $\left[k[u, v, t] /(u v-t) / \mu_{n}\right]$ contributes $1 / n$. We caution the reader that the relation $12 \lambda=\kappa+\delta$ need not hold for $\mathcal{S} / B$. Let $\mathcal{S} \rightarrow S$ be the coarse space and $S \rightarrow B$ the induced morphism. Then we have $\kappa_{S / B}=\kappa_{\mathcal{S} / B}$ and $\lambda_{S / B}=\lambda_{\mathcal{S} / B}$, but $\delta_{\mathcal{S} / B} \neq \delta_{S / B}$ if $\mathcal{S} \neq S$. Indeed, while the orbi-node $\left[k[u, v, t] /(u v-t) / \mu_{n}\right]$ contributes $1 / n$ towards $\delta_{\mathcal{S} / B}$, its coarse space contributes $n$ towards $\delta_{S / B}$. The relation $12 \lambda=$ $\kappa+\delta$ holds for $S / B$.

We now recall the linear algebraic description of triple covers ([14, Theorem 1.1] or [5, Theorem 1.5]). Let $Y$ be an integral scheme and $\phi: X \rightarrow Y$ a finite flat map of degree 3 . We have the exact sequence

$$
0 \rightarrow O_{Y} \rightarrow \phi_{*} O_{X} \rightarrow F \rightarrow 0,
$$

where $F$ is a locally free sheaf of rank 2 on $Y$. The sequence splits by $\frac{1}{3}$ times the trace map $\frac{1}{3} \operatorname{tr}: \phi_{*} O_{X} \rightarrow O_{Y}$. Set $E=F^{\vee}$ and let $p: \mathbf{P} E \rightarrow Y$ be the projectivization. If $\phi$ has Gorenstein fibers then $X$ can be embedded as a Cartier divisor in $\mathbf{P} E$. In that case, the resolution of $O_{X}$ as an $O_{\mathbf{P} E}$ module is given by

$$
0 \rightarrow p^{*} \operatorname{det} E \otimes O_{\mathbf{P} E}(-3) \rightarrow O_{\mathbf{P} E} \rightarrow O_{X} .
$$

Thus, a triple cover of $Y$ is determined by a locally free sheaf $E$ of rank 2 and a section on $\mathbf{P} E$ of the line bundle $O_{\mathbf{P} E}(3) \otimes p^{*} \operatorname{det} E^{\vee}$, or equivalently by a section on $Y$ of the vector bundle $\operatorname{Sym}^{3}(E) \otimes \operatorname{det} E^{\vee}$. Being local on the base, this description holds also if $Y$ is an algebraic stack. 
For the rest of the section, we work with the following setup:

$B$ : a smooth projective curve,

$\pi: \mathcal{S} \rightarrow B:$ a generically smooth, proper family of orbi-nodal curves

$\phi: \mathcal{C} \rightarrow \mathcal{S}:$ a Gorenstein triple cover

$$
E=\left(\phi_{*} O_{\mathcal{C}} / O_{\mathcal{S}}\right)^{\vee} \text {. }
$$

For $a \in \mathbf{Z} / n \mathbf{Z}$, denote by $k(a)$ the line bundle on $B \mu_{n}$ corresponding to the character $\zeta_{n} \mapsto \zeta_{n}^{a}$

Proposition 3.1. In the setup of (3.1), we have

$$
\begin{aligned}
& \kappa_{\mathcal{C} / B}=3 \kappa_{\mathcal{S} / B}+2 c_{1}(E)^{2}+4 c_{1}(E) c_{1}\left(\omega_{\mathcal{S} / B}\right)-3 c_{2}(E), \text { and } \\
& \lambda_{\mathcal{C} / B}=\lambda_{\mathcal{S} / B}+\frac{\kappa_{\mathcal{S} / B}+\delta_{\mathcal{S} / B}}{6}+\frac{c_{1}(E)^{2}}{2}+\frac{c_{1}(E) c_{1}\left(\omega_{\mathcal{S} / B}\right)}{2}-c_{2}(E)+\sum_{\text {Orbi } p \in \mathcal{S}} \chi(p),
\end{aligned}
$$

where $\chi(p)$ is defined as follows. Consider a point $p \in \mathcal{S}$ with stabilizer group $\mu_{n}$. Let the restriction of $E$ to the $B \mu_{n}$ based at $p$ be $k(-a) \oplus k(-b)$. Let $\zeta_{n}$ be a primitive nth root of unity. Then

$$
\chi(p)=\frac{1}{n} \sum_{i=1}^{n-1}\left(\frac{\zeta_{n}^{i a}+\zeta_{n}^{i b}}{2-\zeta_{n}^{i}-\zeta_{n}^{-i}}\right) .
$$

Proof. Let $p: \mathbf{P} E \rightarrow \mathcal{S}$ be the projection, $\xi$ the class of $O_{\mathbf{P} E}(1)$ on $\mathbf{P} E$ and $\xi_{\mathcal{C}}$ its restriction to $\mathcal{C}$, where we think of $\mathcal{C}$ as embedded in $\mathbf{P} E$. Since this embedding is by the relative canonical $\omega_{\mathcal{C} / \mathcal{S}}$, we have

$$
\begin{aligned}
c_{1}\left(\omega_{\mathcal{C} / B}\right) & =c_{1}\left(\omega_{\mathcal{C} / \mathcal{S}}\right)+\phi^{*} c_{1}\left(\omega_{\mathcal{S} / B}\right) \\
& =\xi_{\mathcal{C}}+\phi^{*} c_{1}\left(\omega_{\mathcal{S} / B}\right) .
\end{aligned}
$$

Using $[\mathcal{C}]=3 \xi-p^{*} c_{1}(E)$ and $\operatorname{deg} \phi=3$, we compute

$$
\begin{aligned}
\kappa_{\mathcal{C} / B} & =c_{1}\left(\omega_{\mathcal{C} / B}\right)^{2} \\
& =\xi_{\mathcal{C}}^{2}+2 \xi_{\mathcal{C}} \cdot \phi^{*} c_{1}\left(\omega_{\mathcal{S} / B}\right)+\phi^{*} c_{1}\left(\omega_{\mathcal{S} / B}\right)^{2} \\
& =\left(\xi^{2}+2 \xi \cdot p^{*} c_{1}\left(\omega_{\mathcal{S} / B}\right)\right)\left(3 \xi-p^{*} c_{1}(E)\right)+3 \kappa_{\mathcal{S} / B} \\
& =2 c_{1}(E)^{2}-3 c_{2}(E)+4 c_{1}(E) c_{1}\left(\omega_{\mathcal{S} / B}\right)+3 \kappa_{\mathcal{S} / B}
\end{aligned}
$$

The last step is mere algebraic simplification using $\xi^{2}-p^{*} c_{1}(E) \xi+p^{*} c_{2}(E)=0$.

To compute $\lambda_{\mathcal{C} / B}$, set $\rho=\pi \circ \phi$, and note that in the Grothendieck group of sheaves on $B$, we have

$$
R \rho_{*} O_{\mathcal{C}}=R \pi_{*} O_{\mathcal{S}}+R \pi_{*} E^{\vee}
$$

By taking degrees, we get

$$
\lambda_{\mathcal{C} / B}=\lambda_{\mathcal{S} / B}+c_{1}\left(R \pi_{*} E^{\vee}\right) .
$$

To compute the last term, we use Grothendieck-Riemann-Roch. We must be careful, however, as $\pi: \mathcal{S} \rightarrow B$ is a Deligne-Mumford stack. From [8, Corollary 5.3] and using the notation therein, we have

$$
\operatorname{ch}\left(R \pi_{*} E^{\vee}\right)=\pi_{*}\left(\operatorname{ch}\left(t\left(\frac{f^{*} E^{\vee}}{\lambda_{-1}\left(N_{f}^{*}\right)}\right)\right) \frac{\operatorname{td}(I \mathcal{S})}{\operatorname{td}(B)}\right) .
$$


In this expression, $f: I \mathcal{S} \rightarrow \mathcal{S}$ is the inertia stack, $N_{f}^{*}$ the conormal bundle of $f$, the operator $\lambda_{-1}$ is the alternating sum of wedge powers $\left(1-\wedge^{1}+\wedge^{2}-\cdots\right)$, and the operator $t$ is the 'twisting operator,' which we describe shortly in our context. The inertia stack $I \mathcal{S}$ is the disjoint union

$$
I \mathcal{S}=\mathcal{S} \sqcup \bigsqcup_{\text {Orbi } p \in \mathcal{S}}\left(\mu_{n_{p}} \backslash\{\mathrm{id}\}\right) \times B \mu_{n_{p}},
$$

where $\mu_{n_{p}}$ is the automorphism group at $p$. The conormal bundle $N_{f}$ is trivial on the $\mathcal{S}$ component of $I \mathcal{S}$ and is the cotangent space of $p$ (with the $\mu_{n_{p}}$ action) on the $\{\zeta\} \times B \mu_{n_{p}}$ component, where $\zeta \in \mu_{n_{p}} \backslash\{$ id $\}$. Thus, $\lambda_{-1} N_{f}^{*}$ equals 1 on $\mathcal{S}$ and $(2-k(1)-k(-1))$ on $\{\zeta\} \times B \mu_{n_{p}}$. The twisting operator $t$ acts on the Grothendieck group tensored with $\mathbf{C}$. The action is trivial on the classes of sheaves supported on $\mathcal{S}$, and it sends the class of $k(a)$ supported on $\{\zeta\} \times B \mu_{n_{p}}$ to $\zeta^{a} k(a)$.

Say $E^{\vee}$ splits as $k\left(a_{p}\right) \oplus k\left(b_{p}\right)$ on the $B \mu_{n_{p}}$ at $p$. Then, separating the contributions of the different components of $I \mathcal{S}$ in (3.2) gives

$$
\begin{aligned}
\operatorname{ch}\left(R \pi_{*} E^{\vee}\right)= & \pi_{*}\left(\operatorname{ch}\left(E^{\vee}\right) \operatorname{td}(\mathcal{S} / B)\right)+\sum_{\substack{\text { Orbi } p \in \mathcal{S} \\
1 \neq \zeta \in \mu_{n}}} \frac{1}{n_{p}} \cdot \frac{\zeta^{a_{p}}+\zeta^{b_{p}}}{2-\zeta-\zeta^{-1}} \\
= & \pi_{*}\left(2-c_{1}(E)+\frac{c_{1}(E)^{2}-2 c_{2}(E)}{2}\right) \\
& \left(1-\frac{c_{1}\left(\Omega_{\mathcal{S} / B}\right)}{2}+\frac{c_{1}\left(\Omega_{\mathcal{S} / B}\right)^{2}+c_{2}\left(\Omega_{\mathcal{S} / B}\right)}{12}\right)+\sum_{\text {Orbi } p \in \mathcal{S}} \chi(p),
\end{aligned}
$$

Using $c_{1}\left(\Omega_{\mathcal{S} / B}\right)=c_{1}\left(\omega_{\mathcal{S} / B}\right)$ and $c_{2}\left(\Omega_{\mathcal{S} / B}\right)=\delta_{\mathcal{S} / B}$, we get the desired formula for $\lambda_{\mathcal{C} / B}$.

\section{Sweeping curves}

In this section, we prove the first half of Theorem 1.2 by constructing curves with slope $s_{g}$ that sweep out a Zariski open locus in $\overline{\mathcal{H}}_{g}^{3}$.

4.1. The even genus case. Let $g=2 n-2$. Consider a general pencil of curves in the linear system $|(3, n)|$ on $\mathbf{P}^{1} \times \mathbf{P}^{1}$. This gives a curve $\mathbf{P}^{1} \rightarrow \overline{\mathcal{H}}_{g}^{3}$ and such curves sweep out a Zariski open locus of $\overline{\mathcal{H}}_{g}^{3}$. Expressed differently, let $S=\mathbf{P}^{1} \times \mathbf{P}^{1}$ and denote by $\pi$ the second projection. Denote by $\sigma$ and $F$ the classes of a constant section and a fiber of $\pi$, respectively. Let $E$ be the vector bundle

$$
E=O(n \sigma+F) \oplus O(n \sigma+F) .
$$

Construct a triple cover $\phi: C \rightarrow S$ by letting $C \subset \mathbf{P} E$ be general in the linear system $|3 \xi-\operatorname{det} E|$ where $\xi$ is the class of $O_{\mathbf{P} E}(1)$.

Proposition 4.1. The above construction yields curves that sweep out a Zariski open subset of $\overline{\mathcal{H}}_{g}^{3}$ and have slope $7+6 / g$. 
Proof. Since a generic trigonal curve of genus $g=2 n-2$ embeds in $\mathbf{P}^{1} \times \mathbf{P}^{1}$ as a divisor of class $(3, n)$, we get the first assertion. For the slope, using the formulas of Proposition 3.1, we get

$$
\lambda=g \text { and } \kappa=5 g-6 .
$$

Using $12 \lambda=\kappa+\delta$, we get $\delta=7 g+6$.

4.2. The odd genus case. Let $g=2 n-1$. As in the previous case, let $S=\mathbf{P}^{1} \times \mathbf{P}^{1}$, and denote by $\pi$ the second projection. Denote by $\sigma$ and $F$ the classes of a constant section and a fiber, respectively. Let $E$ be the vector bundle

$$
E=O(n \sigma+F) \oplus O((n+1) \sigma+2 F) .
$$

Construct a triple cover $\phi: C \rightarrow S$ by letting $C \subset \mathbf{P} E$ be general in the linear system $|3 \xi-\operatorname{det} E|$ where $\xi$ is the class of $O_{\mathbf{P} E}(1)$.

Proposition 4.2. The above construction yields curves that sweep out a Zariski open subset of $\overline{\mathcal{H}}_{g}^{3}$ and have slope $7+20 /(3 g+1)$.

Proof. Let $b \in B$ be a point. Denote by $\xi_{b}$ the restriction of $\xi$ to $\mathbf{P} E_{b}$. The main observation is that the map of linear systems

$$
H^{0}\left(O_{\mathbf{P} E}(3 \xi-\operatorname{det} E)\right) \rightarrow H^{0}\left(O_{\mathbf{P} E_{b}}\left(3 \xi_{b}-\operatorname{det} E_{b}\right)\right)
$$

is surjective. Indeed, it suffices to show that

$$
H^{0}\left(\operatorname{Sym}^{3} E \otimes \operatorname{det} E^{\vee}\right) \rightarrow H^{0}\left(\operatorname{Sym}^{3} E_{b} \otimes \operatorname{det} E_{b}^{\vee}\right)
$$

is surjective. The cokernel of this map is $H^{1}\left(\mathrm{Sym}^{3} E \otimes \operatorname{det} E^{\vee} \otimes O(-F)\right)$. Since $\mathrm{Sym}^{3} E \otimes \operatorname{det} E^{\vee}=O((n-1) \sigma) \oplus O(n \sigma+F) \oplus O((n+1) \sigma+2 F) \oplus O((n+2) \sigma+3 F)$, it follows that $H^{1}\left(\operatorname{Sym}^{3}(E) \otimes \operatorname{det} E^{\vee} \otimes O(-F)\right)=0$.

Note that away from a locus of codimension two, a divisor in $\left|3 \xi_{b}-\operatorname{det} E_{b}\right|$ gives a point $\phi: C \rightarrow \mathbf{P}^{1}$ of $\overline{\mathcal{H}}_{g}^{3}$. Coupled with the surjection (4.1), this implies that a general divisor in $|3 \xi-\operatorname{det} E|$ gives a curve in $\overline{\mathcal{H}}_{g}^{3}$. The surjection (4.1) also implies that these curves sweep out a Zariski open subset of $\overline{\mathcal{H}}_{g}^{3}$.

It remains to check the slope. By Proposition 3.1, we get

$$
\lambda=3 n-1 \text { and } \kappa=15 n-15 .
$$

Using $12 \lambda=\kappa+\delta$, we get $\delta=21 n+3$. Hence, the slope is

$$
\frac{\delta}{\lambda}=\frac{21 n+3}{3 n-1}=7+\frac{20}{3 g+1} \text {. }
$$

\section{Effective divisors}

In this section, we prove the second half of Theorem 1.2. The idea is to exhibit an effective divisor with class proportional to $\left(s_{g} \lambda-\delta\right)$. 
5.1. The even genus case. Let $\mu \subset \overline{\mathcal{H}}_{g}^{3}$ be the closure of the locus in $\mathcal{H}_{g}^{3}$ consisting of covers $\left[\phi: C \rightarrow \mathbf{P}^{1}\right]$ for which the rank two bundle $\phi_{*} O_{C} / O_{\mathbf{P}^{1}}$ is unbalanced. An easy dimension count shows that $\mu$ is a divisor [15, Corallary 12.1]. It is called the Maroni divisor. The divisor $\mu$ plus an effective sum of boundary divisors is a positive multiple of $(7+6 / g) \lambda-\delta$. To prove this, we compute the class of $\mu$

$$
\mu=a \lambda-b \delta-c H-\sum c_{i}\left(g_{1}, g_{2}\right) \Delta_{i}\left(g_{1}, g_{2}\right),
$$

by a series of test curve calculations.

Setup of test curve calculations. All the test curves are constructed as follows:

(1) Start with a fibration of rational (orbi)-curves $\pi: \mathcal{S} \rightarrow B$

(2) Pick a sufficiently $\pi$-positive rank two bundle $E$ on $\mathcal{S}$.

(3) Construct $\phi: \mathcal{C} \rightarrow \mathcal{S}$ by picking a generic divisor in $\left|O_{\mathbf{P} E}(3) \otimes \operatorname{det} E^{\vee}\right|$.

Thus, the test-curve is specified by $\mathcal{S} \rightarrow B$ and $E$. In all the test-curves, we have $B=\mathbf{P}^{1}$. The family $\mathcal{S} \rightarrow B$ is one of the following four:

(1) $\mathcal{S}_{0}=\mathbf{P}^{1} \times B$. It is used to compute the coefficients of $\lambda$ and $\delta$.

(2) $\mathcal{S}_{1}$ is obtained by blowing up $\mathbf{P}^{1} \times B$ at a point. It is used to compute the coefficients of $\Delta_{1}\left(g_{1}, g_{2}\right), \Delta_{4}\left(g_{1}, g_{2}\right), \Delta_{6}\left(g_{1}, g_{2}\right)$ and $H$.

(3) $\mathcal{S}_{2}$ is obtained by blowing up $\mathbf{P}^{1} \times B$ along a $k[\epsilon] / \epsilon^{2}$ transverse to a fiber and replacing the resulting $A_{1}$ singularity $k[x, y, t] /\left(x y-t^{2}\right)$ by the orbi-node $\left[k[u, v, t] /(u v-t) / \mu_{2}\right]$. It is used to compute the coefficients of $\Delta_{2}\left(g_{1}, g_{2}\right)$ and $\Delta_{5}\left(g_{1}, g_{2}\right)$.

(4) $\mathcal{S}_{3}$ is obtained by blowing up $\mathbf{P}^{1} \times B$ along a $k[\epsilon] / \epsilon^{3}$ transverse to a fiber and replacing the resulting $A_{2}$ singularity $k[x, y, t] /\left(x y-t^{3}\right)$ by the orbi-node $\left[k[u, v, t] /(u v-t) / \mu_{3}\right]$. It is used to compute the coefficients of $\Delta_{3}\left(g_{1}, g_{2}\right)$.

For all $\mathcal{S}_{i}$, denote by $s$ the preimage of a constant section from $\mathbf{P}^{1} \times B$ not passing through the center of the blowup. For $\mathcal{S}_{i}$ with $i \geq 1$, let $F_{\infty}$ (resp. $F_{0}$ ) be the component of the reducible fiber that does not (resp. does) intersect $s$. Note that

$$
s^{2}=0, \quad F_{0} \cdot F_{\infty}=\frac{1}{i} \text { and } F_{0}^{2}=F_{\infty}^{2}=\frac{-1}{i} .
$$

Also,

$$
\omega_{\mathcal{S}_{0} / B}=-2 s \text { and } \omega_{\mathcal{S}_{i} / B}=-2 s+i F_{\infty} \text { for } i \geq 1 .
$$

Table 1 lists various test-curves, their intersection with the higher boundary divisors and the value of the Residual, defined by

$$
\text { Residual }=(7 g+6) \lambda-g \delta-2(g-3) \mu .
$$

The divisors $\lambda$ and $\delta$ are computed by applying Proposition 3.1 and using $12 \lambda=\kappa+\delta$ for the induced family $\pi: C \rightarrow B$, where $C$ is the coarse space of $\mathcal{C}$. The orbifold correction term $\chi(p)$ in the expression for $\lambda$ is 0 for $\mathcal{S}_{1}, 0$ for $\mathcal{S}_{2}$ and $-2 / 9$ for $\mathcal{S}_{3}$. These curves intersect the higher boundary divisors in general points (Proposition 5.2) and hence the central fiber does not contribute to $\mu$. Note that $\mu$ is 0 on all the testcurves except the second, where it is $(\operatorname{deg} L-\operatorname{deg} M)$.

We take $g=2 n-2$, and let $L$ and $M$ be sufficiently positive line bundles pulled back from $B$. 
TABLE 1. Test-curve calculations for the class of $\mu$ (even $g$ )

\begin{tabular}{|l|l|l|l|}
\hline $\mathcal{S}$ & $E$ & Intersection with & Residual \\
& higher boundary & \\
\hline \hline $\mathcal{S}_{0}$ & $L(n s)^{\oplus 2}$ & - & 0 \\
$\mathcal{S}_{0}$ & Generic extension & - & 0 \\
& $L((n-1) s) \hookrightarrow E \rightarrow M((n+1) s)$. & $\Delta_{1}\left(g_{1}, g_{2}\right)=1$ & $\frac{3}{2} g_{1} g_{2}$ \\
$\mathcal{S}_{1}$ & $L\left(n s-b F_{\infty}\right) \oplus M\left(n s-b F_{\infty}\right)$ & $g_{1}=2(n-b)-2$ & \\
& with $1 \leq b \leq n-1$ & $g_{2}=2 b-2$ & \\
& & $\Delta_{1}\left(g_{1}, g_{2}\right)=1$ & $\frac{1}{2}\left(3 g_{1} g_{2}+g_{1}+g_{2}-1\right)$ \\
$\mathcal{S}_{1}$ & $L\left(n s-b F_{\infty}\right) \oplus M\left(n s-(b-1) F_{\infty}\right)$ & $g_{1}=2(n-b)-1$ & \\
& with $2 \leq b \leq n-1$ & $g_{2}=2 b-3$ & \\
& & $\Delta_{4}\left(g_{1}, g_{2}\right)=1$ & $\frac{1}{2} g_{2}\left(g_{1} g_{2}+g_{2}^{2}+5 g_{1}-1\right)$ \\
$\mathcal{S}_{1}$ & Generic extension $(2 \leq b \leq 2 n-2)$ & \\
& $L\left(n s-b F_{\infty}\right) \hookrightarrow E \rightarrow M(n s)$ & $g_{1}=2 n-b-2$ & \\
& & $g_{2}=b-1$ & \\
$\mathcal{S}_{1}$ & Extension $($ with $n \leq b \leq 2 n-2)$ & $\Delta_{6}\left(g_{1}, g_{2}\right)=1$ & $\frac{1}{2} g_{2}\left(g_{1} g_{2}+g_{2}^{2}+5 g_{1}-g_{2}-6\right)+g$ \\
& $L\left(n s-b F_{\infty}\right) \hookrightarrow E \rightarrow M(n s)$ & $g_{1}=2 n-b-1$ & \\
& such that $E=O \oplus O(2 n-b)$ on $F_{0}$ & $g_{2}=b-1$ & $\frac{1}{2} g(g-2)(g+1)$ \\
$\mathcal{S}_{1}$ & $b=2 n-1$ in the above case. & $H=2$ & \\
$\mathcal{S}_{2}$ & $L\left(n s-b F_{\infty}\right) \oplus M\left(n s-(b-1) F_{\infty}\right)$ & $\Delta_{2}\left(g_{1}, g_{2}\right)=1$ & $3 g_{1} g_{2}$ \\
& & $g_{1}=2 n-b-1$ & \\
& & $g_{2}=b-2$ & \\
$\mathcal{S}_{2}$ & Generic extension $($ with $b$ odd $)$ & $\Delta_{5}\left(g_{1}, g_{2}\right)=1$ & $g_{2}^{3}+g_{1} g_{2}^{2}-2 g_{2}^{2}+4 g_{1} g_{2}-g_{1}-g_{2}$ \\
& $L\left(n s-b F_{\infty}\right) \hookrightarrow E \rightarrow M(n s)$ & $g_{1}=2 n-\frac{b+3}{2}$ & \\
& & $g_{2}=\frac{b-1}{2}$ & \\
$\mathcal{S}_{3}$ & $L\left(n s-b F_{\infty}\right) \oplus M\left(n s-(b-1) F_{\infty}\right)$ & $\Delta_{3}\left(g_{1}, g_{2}\right)=1$ & $\frac{9}{2} g_{1} g_{2}-g_{1}-g_{2}$ \\
& with $b \equiv 2(\bmod 3)$ & $g_{1}=2 n-\frac{2 b+2}{3}$ & \\
& & $g_{2}=\frac{2 b-4}{3}$ & \\
$\mathcal{S}_{3}$ & $L\left(n s-b F_{\infty}\right) \oplus M\left(n s-(b-2) F_{\infty}\right)$ & $\Delta_{3}\left(g_{1}, g_{2}\right)=1$ & $\frac{1}{2}\left(9 g_{1} g_{2}-g_{1}-g_{2}-3\right)$ \\
& with $b \equiv 1(\bmod 3)$ & $g_{1}=2 n-\frac{2 b+1}{3}$ & \\
& & $g_{2}=\frac{2 b-5}{3}$ & \\
& & & \\
& &
\end{tabular}

Remark 5.1. Proposition 3.1 along with $\delta=12 \lambda-\kappa$ gives $\delta$ for the family $\pi: C \rightarrow B$, where $C$ is the coarse space of $\mathcal{C}$. In the following cases, however, the special fiber of $C \rightarrow B$ has unstable rational tails, which must be contracted to get a family of Deligne-Mumford stable curves. We must accordingly adjust the value of $\delta$.

(1) When the special fiber is in $\Delta_{4}\left(g_{1}, g_{2}\right)$, its rational tail, which is a -1 curve, must be contracted. Hence we must subtract 1 from the $\delta$ computed for $\pi: C \rightarrow B$.

(2) When the special fiber is in $\Delta_{5}\left(g_{1}, g_{2}\right)$, its rational tail must be contracted, which removes an $A_{1}$ singularity from $C$. Hence we must subtract 2 from the $\delta$ computed for $\pi: C \rightarrow B$.

(3) When the special fiber is in $\Delta_{6}\left(g_{1}, g_{2}\right)$, its two rational tails, which are both -1 curves, must be contracted. Hence, we must subtract 2 from the $\delta$ computed for $\pi: C \rightarrow B$.

(4) To get a central fiber in $H$ (by setting $b=2 n-1$ in the fifth row), we must contract $F_{\infty}$ and the two rational curves over it. Furthermore, to get a Deligne-Mumford stable model, we must also contract the rational tail on 
the remaining curve. Thus, in total, we must subtract 3 from the $\delta$ computed for $\pi: C \rightarrow B$.

It remains to argue that the curves constructed in Table 1 indeed intersect the boundary divisors in generic points. This is immediate for $\Delta$. The following proposition proves it for the higher boundary divisors.

Proposition 5.2. The special fiber of the family of curves in Table 1 is generic in the boundary divisor indicated in the third column.

Proof. Let $\pi: \mathcal{S}_{i} \rightarrow B$ (for $i \geq 1$ ) and $E$ be as indicated in Table 1 . Let $0 \in B$ correspond to the special fiber. Let $V \subset H^{0}\left(\mathrm{Sym}^{3} E_{0} \otimes \operatorname{det} E_{0}{ }^{\vee}\right)$ be the image of $H^{0}\left(\mathrm{Sym}^{3} E \otimes \operatorname{det} E^{\vee}\right)$. We must prove that the trigonal curves given by generic elements of $V$ make up a dense subset in the corresponding boundary divisor. By choosing $L$ and $M$ sufficiently ample, we are reduced to proving this statement where $V$ is the image of $\pi^{*} \pi_{*}\left(\operatorname{Sym}^{3} E \otimes \operatorname{det} E^{\vee}\right)$ in $H^{0}\left(\operatorname{Sym}^{3} E_{0} \otimes \operatorname{det} E_{0}{ }^{\vee}\right)$. The question is thus local around 0.

Consider a general point $[\phi: C \rightarrow P]$ of $\Delta_{i}\left(g_{1}, g_{2}\right)$, where $P=F_{0} \cup F_{\infty}$ is a balanced orbi-nodal curve (the case of $H$ is handled in this guise by letting $i=6$ and $g_{1}=0$.) Note that line bundles on $P$ have integral degree; rank two bundles split; and for generic $[\phi: C \rightarrow P]$ the splitting of $E$ is indeed as obtained in Table 1 . Let $(B, 0)$ be a germ of a smooth curve and $\pi: \mathcal{S} \rightarrow B$ with $\phi: \mathcal{C} \rightarrow \mathcal{S}$ a smoothing of $\phi: C \rightarrow P$ with smooth $\mathcal{S}$, and whose generic fiber is a smooth, balanced trigonal curve. We show that such a smoothing can be obtained in the curves considered in Table 1.

Let $s: B \rightarrow \mathcal{S}$ be a section intersecting $F_{0}$. Set $E=\left(\phi_{*} O_{\mathcal{C}} / O_{\mathcal{S}}\right)^{\vee}$ and $L=O(n s-$ $\left.b F_{\infty}\right)$, with $n$ and $b$ as in Table 1 . It suffices to prove that $E$ can be expressed as an extension

$$
0 \rightarrow L \rightarrow E \rightarrow M \rightarrow 0,
$$

or equivalently, that there is a nowhere vanishing map $L \rightarrow E$. Set $G=L^{\vee} \otimes E$. For the cases of $\Delta_{1}, \Delta_{2}$, and $\Delta_{3}$, where both branches of $\phi: C \rightarrow P$ are connected, and hence balanced by genericity, we have $H^{1}\left(G_{0}\right)=0$. Therefore, $\pi^{*} \pi_{*} G \rightarrow H^{0}\left(G_{0}\right)$ is surjective. Since $G_{0}$ has a nowhere vanishing section, we conclude that $G$ has a nowhere vanishing section.

The cases of $\Delta_{4}, \Delta_{5}$ and $\Delta_{6}$ are a bit subtle. Think of a section of $G$ as a divisor of class $\sigma=O_{\mathbf{P} G}(1)$ in $\mathbf{P} G$; a nowhere vanishing section corresponds to a divisor that does not contain a fiber of $\mathbf{P} G \rightarrow \mathcal{S}$. On a generic fiber $\mathbf{P}_{t}^{1}$ of $\mathcal{S} \rightarrow B$, the class $\sigma$ is that of a constant section of $\mathbf{P} G_{t} \cong \mathbf{P}^{1} \times \mathbf{P}_{t}^{1}$. Consider a family of divisors $X_{t} \subset \mathbf{P} G_{t}$ in class $\sigma$ and let $X_{0} \subset \mathbf{P} G_{0}$ be the flat limit. Then $X_{0}$ is a Cartier divisor in $\mathbf{P} G_{0}$ of class $\left.(\sigma-a N)\right|_{\mathbf{P} G_{0}}$, where $N=\left.\mathbf{P} G\right|_{F_{\infty}}$ and $a \in \mathbf{Z}$. It suffices to show that $a=0$ and $X_{0}$ does not contain any fibers of $\mathbf{P} G_{0} \rightarrow P$.

Let the preimage of $C \rightarrow P$ over $F_{\infty}$ be $C_{1} \sqcup C_{2}$ where $C_{1} \stackrel{\sim}{\longrightarrow} F_{\infty}$ has degree one and $C_{2} \rightarrow F_{\infty}$ has degree two. Note that $N \cdot C_{2} \neq 0$ but $\sigma \cdot C_{2}=0$. Hence $a=0$ if $X_{0}$ is disjoint from $C_{2}$. Since the intersection number of $X_{0}$ with the fibers of $\left.\mathbf{P} G\right|_{F_{\infty}} \rightarrow F_{\infty}$ is one whereas $C_{2} \rightarrow F_{\infty}$ is a double cover, $X_{0}$ cannot contain $C_{2}$.

Consider the highly singular trigonal curve $C^{\prime} \rightarrow \mathbf{P}^{1}$ obtained by contracting the components $C_{1}$ and $C_{2}$ of $C$ as follows. In the case where $C$ is an orbi-curve, we first pass to the coarse space. The rational component $C_{1}$ is contracted to the (smooth) point of attachment. The hyperelliptic component $C_{2}$ of genus $g_{2}$ is contracted to 
(a)

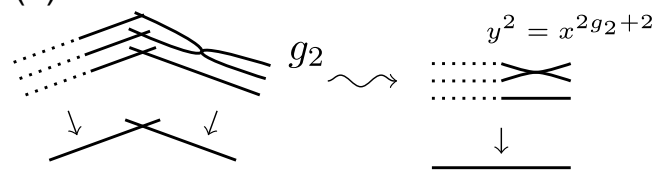

$\Delta_{4}$ or $\Delta_{6}$ (b)

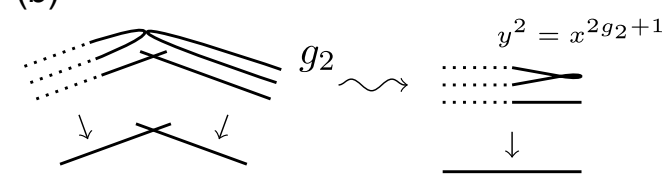

$\Delta_{5}$

Figure 2. Crimping $C \rightarrow P$ to get a singular $C^{\prime} \rightarrow \mathbf{P}^{1}$. (a) $\Delta_{4}$ or $\Delta_{6}$ and (b) $\Delta_{5}$

create a $A_{2 g_{2}+1}$ singularity for $\Delta_{4}$ and $\Delta_{6}$ and a $A_{2 g_{2}}$ singularity for $\Delta_{5}$ (see Figure 2). Note that there is a unique way to carry out such crimping while keeping a map to $\mathbf{P}^{1}$. It is not hard to check that for a generic central fiber $[\phi: C \rightarrow P]$, the resulting $C^{\prime} \rightarrow \mathbf{P}^{1}$ is a balanced triple cover.

Let $\mathcal{S} \rightarrow \mathbf{P}^{1} \times B$ be the contraction of $F_{\infty}$ and $\mathcal{C} \rightarrow \mathcal{C}^{\prime}$ the contraction of the curves in $\mathcal{C}$ over $F_{\infty}$. Then $\mathcal{C}^{\prime} \rightarrow \mathbf{P}^{1} \times B$ is a triple cover; in fact, it is the unique extension to $\mathbf{P}^{1} \times B$ of the cover $\mathcal{C} \backslash \phi^{-1}\left(F_{\infty}\right) \rightarrow \mathcal{S} \backslash F_{\infty}$. The central fiber of $\mathcal{C}^{\prime} \rightarrow \mathbf{P}^{1} \times B$ is $C^{\prime} \rightarrow \mathbf{P}^{1}$, by construction, and the image of $C_{2}$ in $\mathcal{C}^{\prime}$ is the highly singular point (say $x$ ) on $C^{\prime}$. Define the analogues $E^{\prime}$ and $G^{\prime}$ of $E$ and $G$. Consider the flat limit $X_{0}^{\prime}$ of $X_{t}$ in $\mathbf{P} G_{0}^{\prime}$. Since $G^{\prime}$ is balanced, $X_{0}^{\prime}$ is simply a constant section of $\mathbf{P} G_{0}^{\prime} \cong \mathbf{P}^{1} \times \mathbf{P}^{1}$, which will stay away from $x$ for a generic choice of the family $X_{t}$. Then, as $X_{0}$ does not contain $C_{2}$, it follows that $X_{0}$ is disjoint from $C_{2}$. Finally, since $X_{0}^{\prime}$ does not contain any fibers of $\mathbf{P} G_{0}^{\prime} \rightarrow \mathbf{P}^{1}$ and $X_{0}$ does not intersect $C_{2}$, it follows that $X_{0}$ does not contain any fibers of $\mathbf{P} G_{0} \rightarrow P$. The proof is thus complete.

Proposition 5.3. Let $g \geq 4$ be even. In the rational Picard group of $\overline{\mathcal{H}}_{g}^{3}$, we have

$$
2(g-3)[\mu]=(7 g+6) \lambda-g \delta-c H-\sum c_{i}\left(g_{1}, g_{2}\right) \Delta_{i}\left(g_{1}, g_{2}\right),
$$

where $c \geq 0$ and $c_{i} \geq 0$. In particular, a positive multiple of $(7 g+6) \lambda-g \delta$ is equivalent to an effective divisor.

Proof. Intersect both sides of the equation by each of the test-curves in Table 1 . The coefficients $c$ and $c_{i}\left(g_{1}, g_{2}\right)$ are precisely the entries in the "Residual" column. It is easy to verify that these entries are non-negative for the allowed values of $g_{1}, g_{2}$, given $g \geq 4$.

Corollary 5.4. Let $g \geq 4$ be even. Let $B \rightarrow \bar{T}_{g}$ be a curve such that a generic point of $B$ corresponds to a smooth trigonal curve not contained in the image of $\mu$. Then

$$
(7 g+6)(\lambda \cdot B) \geq g(\delta \cdot B) .
$$

Proof. Lift $B \rightarrow \bar{T}_{g}$ to $B \rightarrow \overline{\mathcal{H}}_{g}^{3}$. Then a generic point of $B$ lies in $\mathcal{H}_{g}^{3} \backslash \mu$. We conclude that $B$ intersects non-negatively with $\mu$ and with all the boundary divisors. From Proposition 5.3, we get

$$
(7 g+6)(\lambda \cdot B) \geq g(\delta \cdot B) .
$$


5.2. The odd genus case. Let $\tau \subset \overline{\mathcal{H}}_{g}^{3}$ be the closure of the locus consisting of covers $\left[\phi: C \rightarrow \mathbf{P}^{1}\right]$ for which the scroll $\mathbf{P} E$ is isomorphic to $\mathbf{F}_{1}$ and the image of the embedding $C \hookrightarrow \mathbf{P} E$ is tangent to the directrix, where as usual $E=\left(\phi_{*} O_{C} / O_{\mathbf{P}^{1}}\right)^{\vee}$. Then $\tau$ is a divisor. We call it the tangency divisor. In the odd genus case, it plays the role of the Maroni divisor. The main task is computing its class in the Picard group of $\overline{\mathcal{H}}_{g}^{3}$.

The setup of the test-curve calculations is the same as that in the even genus case (Section 5.1). In particular, we retain the meanings of $B, \mathcal{S}_{i}$ (for $0 \leq i \leq 3$ ), $s, F_{0}$ and $F_{\infty}$. We take $g=2 n-1$ and let $L$ and $M$ be sufficiently ample line bundles pulled back from $B$.

Table 2 lists various test-curves, their intersection with the relevant boundary divisors and the value of the Residual, defined by

$$
\text { Residual }=(21 g+27) \lambda-(3 g+1) \delta-2 \tau .
$$

The divisors $\lambda$ and $\delta$ are computed by applying Proposition 3.1 and using $12 \lambda=\kappa+\delta$ for the induced family $\pi: C \rightarrow B$, where $C$ is the coarse space of $\mathcal{C}$. The divisor $\tau$ is calculated as follows. We pick a section $\sigma: \mathcal{S} \rightarrow \mathbf{P} E$, given by a quotient $E \rightarrow Q$, where $Q$ is a line bundle on $\mathcal{S}$, such that $\sigma$ agrees with the directrix on the generic fiber. We consider the curve $D=\sigma(\mathcal{S}) \cap \mathcal{C}$. The points of $\tau$ on the base $B$ are simply the branch points of $D \rightarrow B$. To compute their number, we note that the class of $D$ on $\mathcal{S}$ is given by

$$
\begin{aligned}
{[D] } & =\sigma^{*}[\mathcal{C}] \\
& =\sigma^{*}\left(3 \xi-c_{1}(E)\right)=3 c_{1}(Q)-c_{1}(E) .
\end{aligned}
$$

By adjunction, we get

$$
\begin{aligned}
\tau & =c_{1}\left(\omega_{D / B}\right) \\
& =\left(3 c_{1}(Q)-c_{1}(E)\right) \cdot\left(3 c_{1}(Q)-c_{1}(E)+c_{1}\left(\omega_{\mathcal{S} / B}\right)\right) .
\end{aligned}
$$

The curves in Table 2 intersect the higher boundary divisors in general points. Hence, the special fiber does not contribute to $\tau$. The formula (5.1), however, includes a contribution from the special fiber for some test-curves, which we must correct. We comment upon these adjustments in Remark 5.5. In Table 2, the role of $Q$ is always played by the twist of $M$.

Remark 5.5. In the following cases, we have to make adjustments to $\delta$ and $\tau$. The adjustments for $\delta$ are for the same reasons as in the case of even genus (Remark 5.1). For the adjustments to $\tau$, recall the notation in (5.1): $\sigma: \mathcal{S} \rightarrow \mathbf{P} E$ is given by a map from $E$ to a twist of $M$; we set $D=\sigma^{*}(\mathcal{C})$ and get $\tau$ by counting the number of branch points of $D \rightarrow B$ using adjunction.

(1) When the special fiber is in $\Delta_{4}\left(g_{1}, g_{2}\right)$, the curve $D$ includes $F_{\infty}$ as a connected component with multiplicity $b$. This unnecessarily contributes $-b-b^{2}$ in the adjunction formula, which we must correct. Also, we must subtract 1 from the $\delta$ counted for $\pi: C \rightarrow B$.

(2) When the special fiber is in $\Delta_{5}\left(g_{1}, g_{2}\right)$, the curve $D$ includes includes $F_{\infty}$ as a connected component with multiplicity $b$. This unnecessarily contributes $-b-b^{2}$ in the adjunction formula, which we must correct. Also, we must subtract 2 from the $\delta$ counted for $\pi: C \rightarrow B$. 
TABLE 2. Test-curve calculations for the class of $\tau$ (odd $g$ )

\begin{tabular}{|c|c|c|c|}
\hline $\mathcal{S}$ & $E$ & $\begin{array}{l}\text { Intersection with } \\
\text { higher boundary }\end{array}$ & Residual \\
\hline $\mathcal{S}_{0}$ & $E=L((n+1) s) \oplus M(n s)$ & - & 0 \\
\hline $\mathcal{S}_{1}$ & $\begin{array}{l}L\left((n+1) s-b F_{\infty}\right) \oplus M\left(n s-b F_{\infty}\right) \\
\text { with } 1 \leq b \leq n-1\end{array}$ & $\begin{array}{l}\Delta_{1}\left(g_{1}, g_{2}\right)=1 \\
g_{1}=2(n-b)-1 \\
g_{2}=2 b-2\end{array}$ & $\frac{3}{2} g_{2}\left(3 g_{1}+1\right)$ \\
\hline $\mathcal{S}_{1}$ & $\begin{array}{l}\text { Generic extension }(2 \leq b \leq 2 n-1) \\
L\left((n+1) s-b F_{\infty}\right) \hookrightarrow E \rightarrow M(n s)\end{array}$ & $\begin{array}{l}\Delta_{4}\left(g_{1}, g_{2}\right)=1 \\
g_{1}=2 n-b-1 \\
g_{2}=b-1\end{array}$ & $\frac{1}{2} g_{2}\left(3 g_{1} g_{2}+3 g_{2}^{2}+15 g_{1}-g_{2}\right)$ \\
\hline $\mathcal{S}_{1}$ & $\begin{array}{l}\text { Extension (with } n+1 \leq b \leq 2 n-1) \\
L\left((n+1) s-b F_{\infty}\right) \hookrightarrow E \rightarrow M(n s) \\
\text { such that } E=O \oplus O(2 n-b+1) \text { on } \\
F_{0}\end{array}$ & $\begin{array}{l}\Delta_{6}\left(g_{1}, g_{2}\right)=1 \\
g_{1}=2 n-b \\
g_{2}=b-1\end{array}$ & $\begin{array}{l}\frac{1}{2} g_{2}\left(3 g_{1} g_{2}+3 g_{2}^{2}+15 g_{1}-4 g_{2}-15\right)+ \\
3 g+1\end{array}$ \\
\hline $\mathcal{S}_{1}$ & $b=2 n$ in the above case. & $H=2$ & $\frac{1}{2}(3 g-4)(g-1)(g+1)$ \\
\hline $\mathcal{S}_{2}$ & $L\left((n+1) s-b F_{\infty}\right) \oplus M\left(n s-(b-1) F_{\infty}\right)$ & $\Delta_{2}\left(g_{1}, g_{2}\right)=1$ & 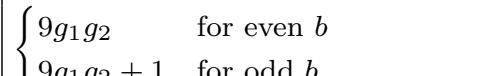 \\
\hline & & & $\left(9 g_{1} g_{2}+1\right.$ for $\mathrm{o}$ \\
\hline $\mathcal{S}_{2}$ & $\begin{array}{l}\text { Generic extension (with } b \text { odd) } \\
L\left((n+1) s-b F_{\infty}\right) \hookrightarrow E \rightarrow M(n s)\end{array}$ & $\begin{array}{l}\Delta_{5}\left(g_{1}, g_{2}\right)=1 \\
g_{1}=2 n-\frac{b+1}{2} \\
g_{2}=\frac{b-1}{2}\end{array}$ & $\begin{array}{l}g_{2}\left(3 g_{2}^{2}+3 g_{1} g_{2}+12 g_{1}-7 g_{2}+4\right)- \\
3 g-1\end{array}$ \\
\hline $\mathcal{S}_{3}$ & $\begin{array}{l}L\left((n+1) s-b F_{\infty}\right) \oplus M\left(n s-(b-1) F_{\infty}\right) \\
\text { with } b \equiv 2(\bmod 3)\end{array}$ & $\begin{array}{l}\Delta_{3}\left(g_{1}, g_{2}\right)=1 \\
g_{1}=2 n-\frac{2 b-1}{3} \\
g_{2}=\frac{2 b-4}{3}\end{array}$ & $\frac{3}{2}\left(9 g_{1} g_{2}-2 g_{1}-g_{2}\right)-1$ \\
\hline $\mathcal{S}_{3}$ & $\begin{array}{l}L\left((n+1) s-b F_{\infty}\right) \oplus M\left(n s-(b-2) F_{\infty}\right) \\
\text { with } b \equiv 1(\bmod 3)\end{array}$ & $\begin{array}{l}\Delta_{3}\left(g_{1}, g_{2}\right)=1 \\
g_{1}=2 n-\frac{2 b-2}{3} \\
g_{2}=\frac{2 b-5}{3}\end{array}$ & $\frac{3}{2}\left(9 g_{1} g_{2}-g_{1}-2 g_{2}\right)-1$ \\
\hline
\end{tabular}

(3) When the special fiber is in $\Delta_{6}\left(g_{1}, g_{2}\right)$, the curve $D$ includes $F_{\infty}$ as a connected component with multiplicity $b$. This unnecessarily contributes $-b-b^{2}$ in the adjunction formula, which we must correct. Also, we must subtract 2 from the $\delta$ counted for $\pi: C \rightarrow B$.

(4) When the central fiber is in $H$ (in the guise of $\Delta_{6}\left(g_{1}, 0\right)$ in the fifth row), the curve $D$ includes $F_{\infty}$ as a connected component with multiplicity $b$. This unnecessarily contributes $-b-b^{2}$ in the adjunction formula, which we must correct. Also, we must subtract 3 from the $\delta$ counted for $\pi: C \rightarrow B$.

(5) When the central fiber is in $\Delta_{2}$ and $g_{2}$ is odd, the curve $D$ passes through the orbifold point of $\mathcal{S}_{2}$. Although the coarse space of $D$ is unramified over $B$ here, the orbifold point unnecessarily contributes $1 / 2$ to the adjunction formula, which we must correct.

Proposition 5.6. The central fiber of the family of curves in Table 2 is generic in the boundary divisor indicated in the third column.

Proof. The proof is the same as that of Proposition 5.2, with one modification in the second half dealing with $\Delta_{4}, \Delta_{5}$ and $\Delta_{6}$. Consider the highly singular trigonal curve $C^{\prime} \rightarrow \mathbf{P}^{1}$ of genus $g$ obtained by contracting the rational and the hyperelliptic tail of the special fiber $\phi: C \rightarrow P$. To ensure the genericity of the central fiber $\phi: C \rightarrow P$, we must not only assume that $C^{\prime} \rightarrow \mathbf{P}^{1}$ is balanced, but also that the $A_{k}$ singularity 
of $C^{\prime}$ lies away from the directrix in the embedding $C^{\prime} \hookrightarrow \mathbf{F}_{1}$. The rest of the proof is almost verbatim.

We readily deduce the analogues of Proposition 5.3 and Corollary 5.4.

Proposition 5.7. Let $g \geq 5$ be odd. In the rational Picard group of $\overline{\mathcal{H}}_{g}^{3}$, we have

$$
2[\tau]=(21 g+27) \lambda-(3 g+1) \delta-c H-\sum c_{i}\left(g_{1}, g_{2}\right) \Delta_{i}\left(g_{1}, g_{2}\right),
$$

where $c \geq 0$ and $c_{i} \geq 0$. In particular, a positive multiple of $(21 g+27) \lambda-(3 g+1) \delta$ is equivalent to an effective divisor.

Corollary 5.8. Let $g \geq 5$ be odd. Let $B \rightarrow \bar{T}_{g}$ be a curve such that a generic point of $B$ corresponds to a smooth trigonal curve not contained in the image of $\tau$. Then

$$
(21 g+27)(\lambda \cdot B) \geq(3 g+1)(\delta \cdot B) .
$$

\section{Acknowledgments}

The work in this paper began after conversations with Maksym Fedorchuk when the authors were graduate students of Joe Harris. We thank both of them for their kindness and appreciation. During the preparation of the article, we learned about the results of Beorchia and Zucconi [3] about similar questions. We thank them for sharing their manuscripts and thoughts.

\section{References}

[1] D. Abramovich, A. Corti and A. Vistoli, Twisted bundles and admissible covers, Comm. Algebra 31(8) (2003), 3547-3618.

[2] M.Á. Barja and L. Stoppino, Slopes of trigonal fibred surfaces and of higher dimensional fibrations, Ann. Sci. Norm. Super. Pisa Cl. Sci. (5) 8(4) (2009), 647-658.

[3] V. Beorchia and F. Zucconi, On the slope conjecture for the fourgonal locus in $\bar{M}_{g}$, arXiv:1209.3571 [math.AG], 2012.

[4] M. Bolognesi and A. Vistoli, Stacks of trigonal curves, Trans. Amer. Math. Soc. 364(7) (2012), 3365-3393.

[5] G. Casnati and T. Ekedahl, Covers of algebraic varieties I. A general structure theorem, covers of degree 3, 4 and Enriques' surfaces, J. Algebr. Geom. 5 (1996), 439-460.

[6] M. Cornalba and J. Harris, Divisor classes associated to families of stable varieties, with applications to the moduli space of curves, Ann. Sci. École Norm. Sup. (4) 21(3) (1988), 455-475.

[7] A. Deopurkar, Compactifications of Hurwitz Spaces, Int. Math. Res. Not. IMRN (2013) doi:10.1093/irnrn/rnt060.

[8] D. Edidin, Riemann-Roch for Deligne-Mumford stacks, in a celebration of algebraic geometry, Vol. 18 of Clay Math. Proc., 241-266, Amer. Math. Soc., Providence, RI (2013).

[9] D. Eisenbud and J. Harris, The Kodaira dimension of the moduli space of curves of genus $\geq 23$, Invent. Math. 90(2) (1987), 359-387.

[10] M. Fedorchuk and D. Jensen, Stability of 2nd Hilbert Points of Canonical Curves, Int. Math. Res. Not. 2013(2) (2013), 5270-5287.

[11] M. Fedorchuk and D.I. Smyth, Alternate Compactifications of Moduli Spaces of Curves, (G. Farkas and I. Morrison, eds.), Handbook of Moduli: Volume I, no. 24 in 'Advanced Lecture in Mathematics (ALM)', International Press, Somerville, MA, 2013, 331-414.

[12] J. Harris and D. Mumford, On the Kodaira dimension of the moduli space of curves, Invent. Math. 67(1) (1982), 23-88.

[13] B. Hassett, Moduli spaces of weighted pointed stable curves, Adv. Math. 173(2) (2003), $316-352$.

[14] R. Miranda, Triple covers in algebraic geometry, Amer. J. Math. 107(5) (1985), 1123-1158.

[15] Z.E. Stankova-Frenkel, Moduli of trigonal curves, J. Algebr. Geom. 9(4) (2000), 607-662. 
Department of Mathematics, Columbia University, 2990 Broadway, New York, NY 10027, USA

E-mail address: anandrd@math.columbia.edu

Department of Mathematics, Boston College, Carney Hall, Chestnut Hill, Ma 02467, USA

E-mail address: anand.patel@bc.edu 